\title{
Study on Improving the Network Life Time Maximazation for Wireless Sensor Network using Cross Layer Approach
}

\author{
Rajiv R Bhandari, K Rajasekhar \\ Department of Computer Engineering, K L University, Vijaywada, India
}

\begin{tabular}{l} 
Article Info \\
\hline Article history: \\
Received May 9, 2016 \\
Revised Jul 18, 2016 \\
Accepted Aug 1, 2016 \\
\hline
\end{tabular}

Keyword:

Adhoc on demand distance

Media access control

Point cordination function

Transmission control protocol

Vector routing

Wireless sensor network

\begin{abstract}
In recent the espousal of Wireless Sensor Networks has been broadly augmented in numerous divisions. Battery operated Sensor nodes in the wireless network accomplish main task of capturing and responding to the surroundings. The lifetime of the network depends on the energy consumption of the sensor nodes. This paper contributes the survey on how the energy consumption should be managed for maximizing the life time of network and how to improve the efficiency of Network by using Cross layer architecture. The traditional MAC Layer, Network Layer \& Transport for WLAN having their own downsides just by modifying those we can achieve the network life time maximization goal. This paper represents analytical study for Energy efficiency by modifying Scheduling algorithm, by modifying traditional AODV routing algorithm for efficient packet transmission and by effectively using TCP for End to End Delivery of Data.
\end{abstract}

Copyright () 2016 Institute of Advanced Engineering and Science. All rights reserved.

\section{Corresponding Author:}

Rajiv Ramesh Bhandari, Departement of Computer Engineering, K L University, Green Fields, Vaddeswaram, Guntur, Andhra Pradesh, 522 502, India. Email: rajivrbhandari@gmail.com

\section{INTRODUCTION}

Now a Days Most of the Digital devices such as Mobile Phones, Kindles, i-phone, i-pads are equipped with WLAN Interfaces. WLAN Interface allows user to access Internet Application Services in Digital Devices. WLAN is equipped with multiple protocols to access channel bandwidth unfortunately all are energy Consuming. Minimizing energy consumption of WLAN interface is an important design issue in Wireless sensor Network [1].

In Routing the packets are routed among the Network. In WSN the role of routing protocol is to find the optimal route among the nodes in dynamic topology using available resources. Due to dense Network and changing locations standard routing protocols consumes a lot of Energy to route a data from source to destination.

The Major task of transport layer is to assure reliable packet delivery for end to end transmission \& congestion control mechanism to regulate the amount of traffic flow within WSN to avoid Packet loss. Transport layer is having multiple techniques for controlling traffic \& avoiding congestion but these techniques consumes lot of power \& resources [2].

Rest of the paper is organized as follows Section 2 represents related work in MAC layer, Network layer \& Transport layer Section 3 represents proposed work Section 4 represents conclusion. The Original 802.11 Medium Access Control specifies that access point might announce a CFP \& CP in which all station must access the WLAN Channel. The MAC having two access channel Mechanism: Point Coordination Function (PCF) \& Distributed Coordination Function (DFC). Station can operate either in Active Mode or Power Saving Mode. Station Must Stay awake to access WLAN channel and receive and transmit packets in Active Mode. The other mode defined in 802.11 is Power Saving Mode (PSA), in which the packets are not 
transmitted or received, also known as sleep mode. During this mode maximum hardware components are switched off and it does not attend to WLAN channel [3].

\section{RELATED WORK}

Raul Palacios et al. has proposed an energy efficient MAC protocol, which is based on infrastructure WLAN. The modified point and distributed coordination function (PCF/DCF) schemes is aimed to increase energy efficiency using bidirectional data packet exchange. According to data traffic at the destination either standard or bidirectional, the operating modes are classified in to two categories. Standard DCF is preferred when the destination has no packet to exchange. In bidirectional mode along with bidirectional packets to exchange, the duration of entire bidirectional channel is exchanged and inserted in the duration field of CTS frame. In modified PCF packet transmission between access point and stations the poll and null frames are decreased which will provide better result in consumption of channels. Using the modified PCF and DCF the advance in terms of throughput and energy saving is notified. The PCF/DCF can also be modified when stations have no packets to send, this will lead to another dimension of research [4].

Thomas Cuzanauskas et al. has presented activity depended power allocation algorithm for IEEE 802.11 networks. Point Coordination Function mechanism is modified with power control functionality based on game-theoretic foundations. In the proposed theory uniform priority is assigned to every station which are connected to particular access point. It is observed that if the numbers of polls without any data send by specific station is grater then some predefined value then station will be considered as ideal state. The sleep period is continuing for some threshold value. By using this strategy appropriate power allocation is done in which high power is diverted to active state and low power is diverted to idle state. The Power Allocation Strategy for Active Station does not create more intervention to legacy Devices [5].

Xuanming Dong et al. has proposed polling algorithm is well-matched with the IEEE 802.11 standard polling list is conceptually splitted into station lists with different priorities to increase the computation efficiency. All stations with the same priority are placed in the same list. The aim of this scheduling algorithm is to avoid polling idle stations and assign more polling slots to stations with heavy traffic. The frequency for polling idle stations is less which means that it avoids polling of idle state. Point coordinator with long duration is use to poll idle station. The priority of each station is updated regularly based on the recent poll feedback from the station, using the Additive Increase/Multiplicative Decrease algorithm. The simulation results show that our proposed polling algorithm improves the performance of wireless LANs in terms of the successful poll rate and the aggregate throughput of access point.

M. Ambigavathi and Dr. Shridharan have modified AODV routing protocol which classifies traffic based on critical data. They have substituted the FIFO scheduling algorithm of AODV with priority queuing algorithm. The algorithm follows two strategies Pre-emption for critical, emergency or on demand data and non-pre-emption for normal data. Based on the priority of data in the queue the packets will be selected for data transmission. Using this modified algorithm the destination will receive the emergency, critical or on demand data in less time period [6].

Dr. R. Dhaya et al. has proposed finest power efficient steering algorithm for WSN which uses the neighboring node power level. IT maintains the forwarder list of neighboring nodes. The node will add into transmission path only if the Neighboring nodes in the forwarder list should have finest energy for efficient transmission else alternative path will be selected. This algorithm increases throughput of network and reduces the consumption of energy [7].

Tong Liu et al. has introduced fixed delay mechanism instead of random delay in AODV. The delay function inversely related with rest energy level. To modify the forward function with fixed delay, it calculates the number of nodes before the RREQ will arrive. These nodes are assessed and their left over energy is sum up which is known as Energy_total and added into RREQ. The lifetime of network has been increased by leveling the energy consumed by nodes [8].

\section{PROPOSED SOLUTION}

\subsection{MAC Layer Modification for sleep scheduling}

We propose scheduling using PCF mode of 802.11 protocols at each node. PCF mode in 802.11 protocols provides facility of channel sharing in terms of time slot. Each of the nodes is allotted particular contention free period for channel utilization by the repeater node during which other nodes are kept in wait state. When particular node is having more traffic load when acting as repeater for multiple source nodes simultaneously, the contention free period allotted for this node should be increased during which maximum amount of packets are possible to be transmitted back to back. Due to this the packets kept in queues for waiting will have to wait only for shorter period which further increases the fast and reliable delivery of 
packets by avoiding occurrence of queue overflows respective packet drops. During this contention free period other nodes which are in wait state can be set in sleep mode to save their energies. This sleep scheduling can be done by sending beacon packet from node having more traffic to each neighbor node, acting as source of packets, excluding one node from set of nodes acting as source of packets, at a time, to start their wait timer. The excluded node will keep on sending the packets till the contention free period allotted expires. As soon as this wait timer in sleeping nodes expires, node will become active and start sending packets to its respective next hop and at the same time previously active node will get into sleep node by setting its timer. Figure 1 indicates the frame scheduling mechanism in PCF mode of 802.11 MAC.

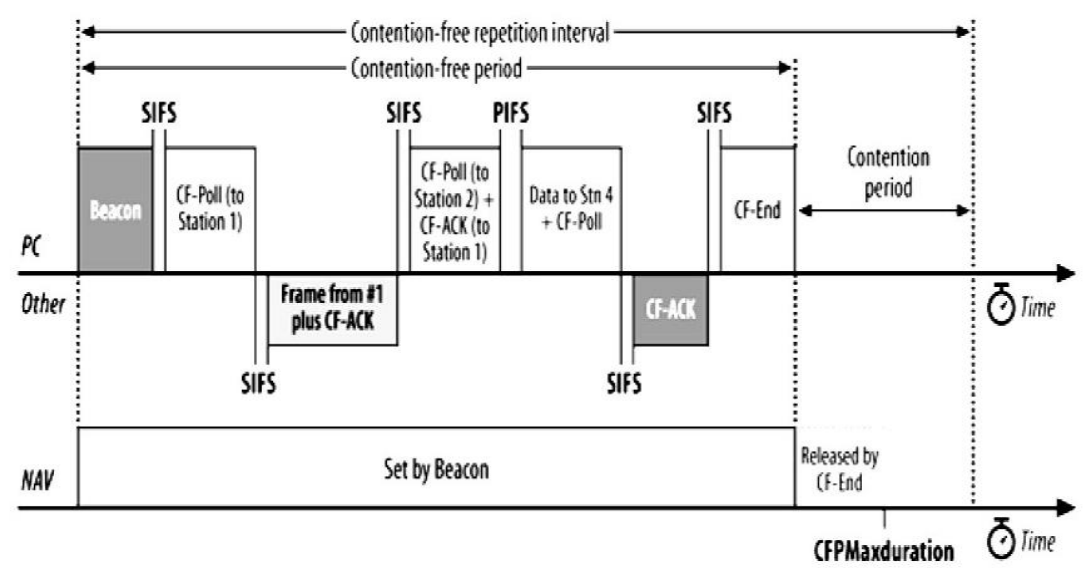

Figure 1. Frame Scheduling in PCF Mode of 802.11 MAC

The time slot allotted will be dependent on the emission of traffic load in the network by each node. If particular node emits larger packets in the network then to prevent packet loss for this node contention free period allotted to this node is slightly increased than that of default values.

Due to this increased time slot, the node will emit more packets in the network than keeping them in queue which will ultimately prevent overflows of the queues. This will lead to packet aggregation mechanism as indicated in Figure 1. This in turn will ultimately increase throughput and packet delivery ratios. As successful packet deliveries are obtained loss of energy for lost packets will be decreased. At the sometime as slight increment will lead to more sleep timer for nodes sleep mode. This will again elongate the life of the node. As sleep timer is increased more packets will be queued and when such node will become active it will try delivering maximum packets in contention free period. Due to this, such node will be identified as node with more traffic hence contention free period allotted to this node will again gets increased and in turn this will be again beneficial as like earlier node. The total number of packets emitted before modification can be indicated by the equation below:

$$
\text { Ptotal }=\sum_{i=t}^{i=T} P i
$$

where, $\mathrm{Pi}$ is the total number of packets emitted by the node during the time period ' $\mathrm{t}$ ' to ' $\mathrm{T}$ '. The equation for total number of packets emitted after modification can be indicate as below:

$$
\text { Ptotal }=\sum_{i=t}^{i=T+\Gamma} P i
$$

where $\Gamma$ indicates the increased time slot.

The sleep scheduling can be well understood from the Figure 2. Node c has got time slot as node c has more traffic load and further node $\mathrm{d}$ and node e are scheduled to send packets. The timed diagram indicates the waking up time of each node. Figure 3 indicates the contention free period allotted for each station during which only one station is allotted time slot for communication. This time slot is increased as indicated in Figure 4. 


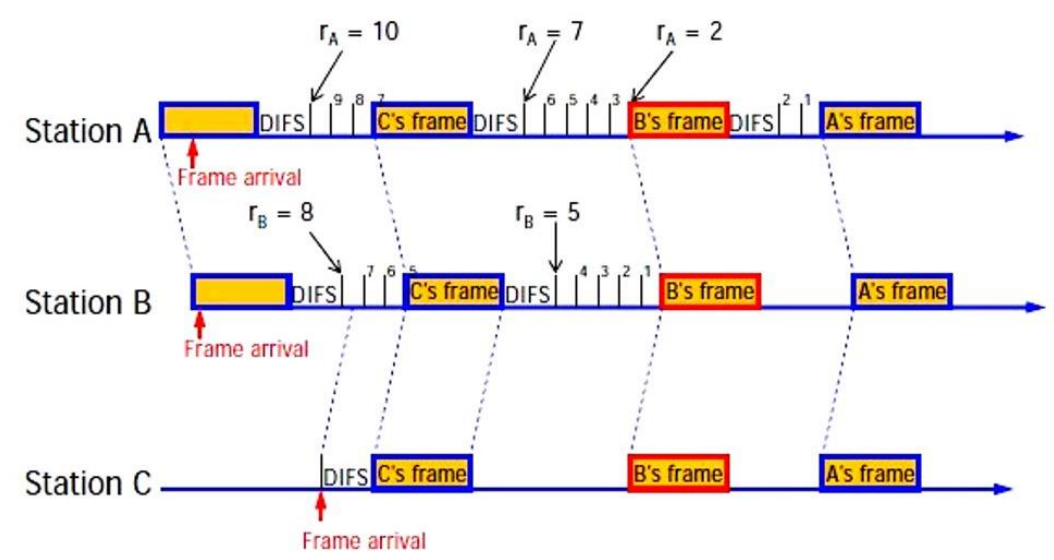

Figure 2. Mobile Stations Allotted Time Slots for Accessing Channel During Sleep Scheduling

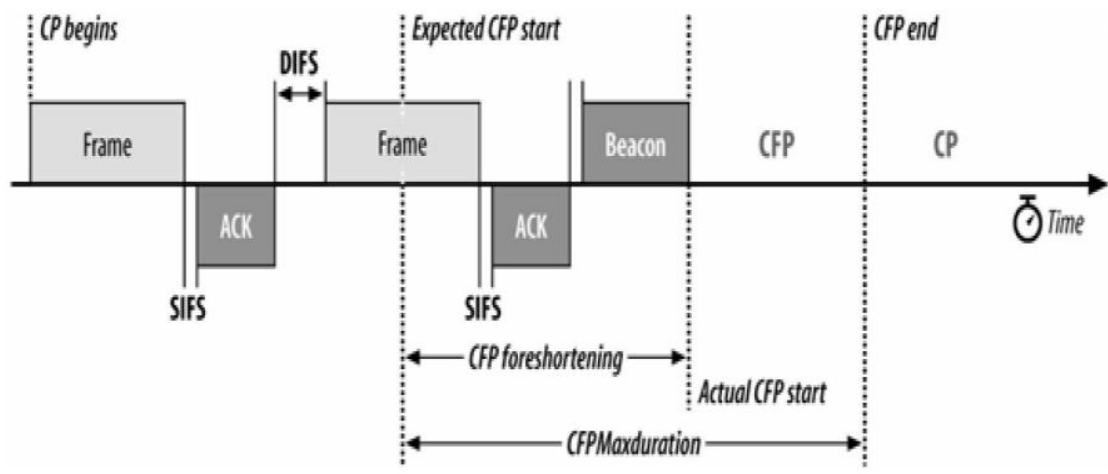

Figure 3. Existing Interframe Time Slots Allotted for Different Stations

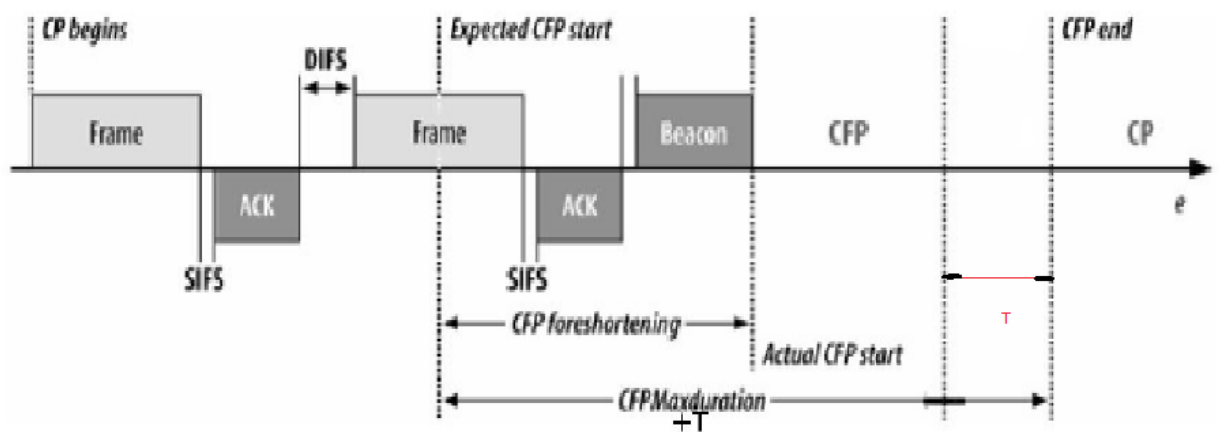

Figure 4. Modified Interframe Time Slots Allotted for Different Stations

In this way Modified PCF mode along with sleep scheduling in 802.11 MAC protocol will lead to increased energy efficiency along with throughput and successful packet delivery ratio in turn increased life time of the network.

\subsection{Modification in AODV protocol to select reliable paths}

To design a energy-efficient routing protocol, we first describe an energy model to measure the energy-factor of the nodes and then we propose a Node Energy Aware Protocol for Energy-Efficient Routing. To understand the mechanism we consider the example of WSN with 49 nodes in the network.

The partial mechanism of RREQ broadcast of AODV can be understood from adhoc network consisting of 49 nodes as shown in figure1. This network is of adhoc wireless network into which Network layer protocol 
is AODV and Datalink and Physical layer is of 802.11 wlan. According to AODV protocol RREQ Message will be broadcasted from source node 43 for searching destination node 7 . This broadcasted message will again be broadcasted further by intermediate nodes in search of destination node 7. RREQ Message Mechanism is shows in Figure 5.

For RREQ message appropriate destination node 7 will only send RREP to the source node 43 which is of unicast type. RREP mechanism can be well understood from Figure 6.

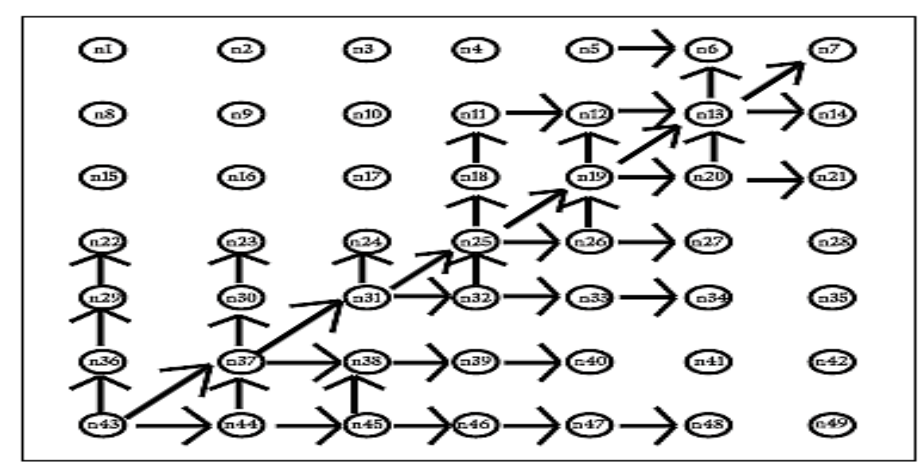

Figure 5. RREQ Message Mechanism

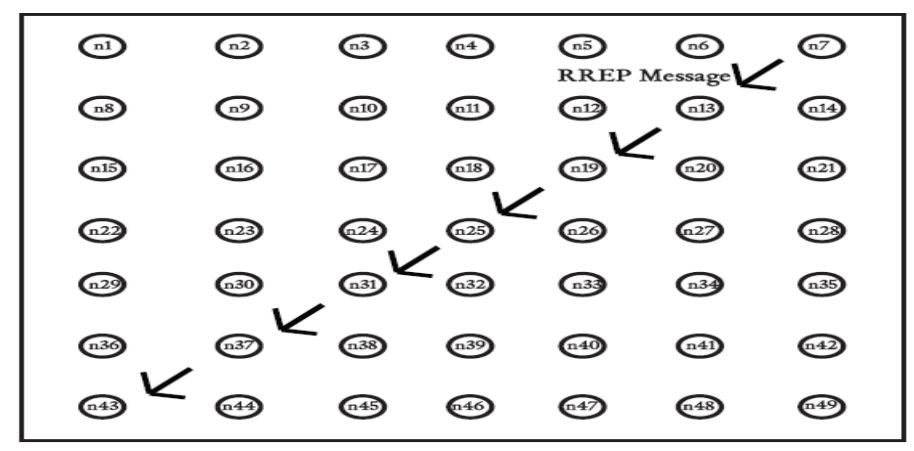

Figure 6. RREP Message Mechanism

Basic AODV message flow process in which due to death of node no. 28 no route error message by 16 to 49 as shown in Figure 7.

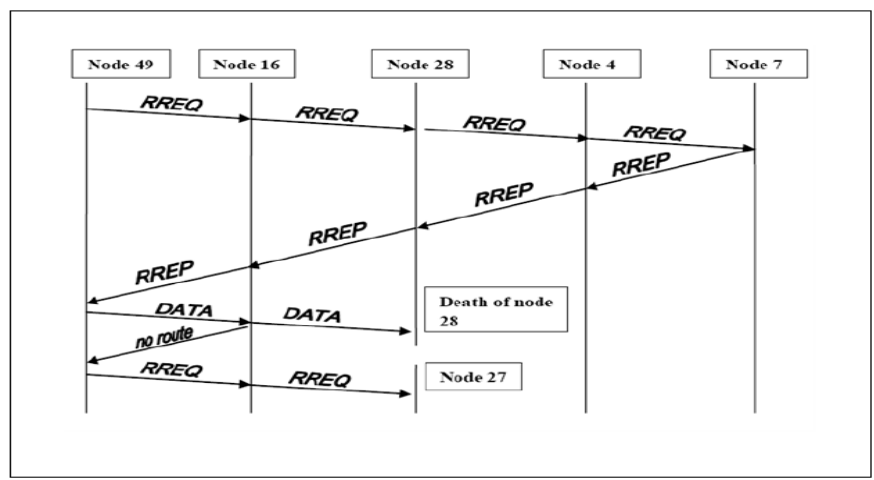

Figure 7. Basic AODV Root Finding Mechanism 
Modified AODV which decreases chances of no route error \& hence can increases reliability. Ultimate outcome is due to avoidance repetitive route establishment energy-efficiency increases as shown in Figure 8.

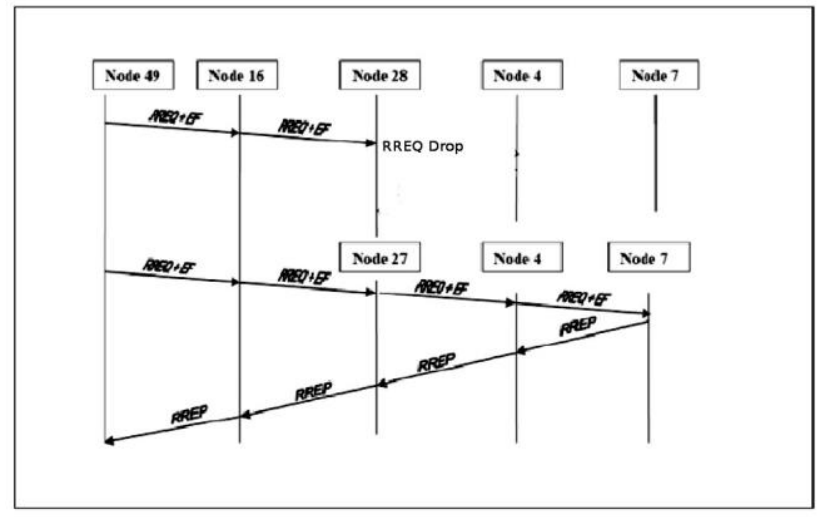

Figure 8. Modified AODV root finding mechanism

\subsection{Flow control and packet delivery scheduling in TCP using MAC layer information}

MAC layer is responsible for allowing node to make use of channel at specific time interval by establishing coordinating activity associated with all the neighboring and in route nodes. The in route nodes are involved as per AODV routing protocol mechanism. Regarding traffic load accurate information can be used when 802.11 involves carrier sense multiple access (CSMA) mechanism to avoid congestion and collisions on the channel. This MAC layer information becomes utilizable to enhance throughput and successful packet deliveries in TCP protocol.

When event occurs in WSN sensor node starts generating packets and emitting them in network. The packet emission rate can be controlled in TCP on weighted priority algorithm. MAC layer can provide link load information to TCP layer by using which link scheduling can be implemented by assigning weight to each link. The weight assigned to packets sequence will be responsible for packets priority to deliver. This will avoid flooding effects and loss of packets in the network thereby increasing successful packet delivery ratios.

The mathematical illustration of weight assignment is indicated as below. We assign weight $q(t)$ to each link at time instant ' $t$ '. The algorithm picks up schedules $(t)$ to deliver the packet such that,

$\mathrm{S}(\mathrm{t})=\max \left(\right.$ selected link ' $\mathrm{l}$ ' out of ' $\mathrm{M}$ ' links $\left.\sum \mathrm{q}(\mathrm{t})\right)$

The equation indicates the maximum time schedule $\mathrm{s}(\mathrm{t})$ picked up for packet delivery on link ' $\mathrm{l}$ ' from ' $M$ ' number of links, assigned each of it weight $q(t)$ at time instant ' $t$ '. We use discrete time model in which packet arrival and departure order is specified as below:

a. At the beginning of each time slot MAC layer delivers the set of packets to each scheduled link according to decision made by scheduling algorithm.

b. For new set of packets arrived TCP establishes new connection with initial pre assigned congestion window size.

c. For each TCP connection if congestion window is not full packets are sent to MAC layer for delivery from transport layer until window size is fully used and all the packets are delivered

When packets are not assigned priority as per weight of the link, those will be delivered as per basic mechanism of TCP protocol. In, weighted and un-weighted, both cases packets will be delivered on FIFO operations mechanism. The sequence assigned to each packet will start from 1 number onwards starting first packet arrival and as soon as first packet is delivered each of upcoming packets will start again numbered from 1 in sequential fashion.

\section{CONCLUSION}

Theoretically we have shown that modification in transport layer mechanism to control rate depending upon MAC layer information we can control the flooding effects in the network and hence ultimately it improves packet delivery ratio thereby reducing packets drop ratio which ultimately shows increment in energy efficiency. Modifying the AODV protocol to select energy efficient nodes in the route 
leads to avoiding inclusion of nodes with less energies and hence avoiding link recovery and re route establishment procedure thereby reducing such energy requirement. Hence it increases energy efficiency. We have proposed to use PCF mode of 802.11 MAC in which we have modified the time slot allotment for channel sharing and sleep scheduling which increases energy saving in each node. The whole process results in increment in life time.

\section{REFERENCES}

[1] Tsao S. L. and Huang C. H., "A survey of energy efficient MAC protocols for IEEE 802.11 WLAN," Computer Communications, vol/issue: 34(1), pp. 54-67, 2011.

[2] Sirsikar S. and Anavatti S., "Issues of Data Aggregation Methods in Wireless Sensor Network: A Survey," Procedia Computer Science, vol. 49, pp. 194-201, 2015.

[3] Maleh Y. and Ezzati A., "Performance analysis of routing protocols for wireless sensor networks," in Information Science and Technology (CIST), 2014 Third IEEE International Colloquium, pp. 420-424, 2014.

[4] Palacios R., et al., "An energy-efficient MAC protocol for infrastructure WLAN based on modified PCF/DCF access schemes using a bidirectional data packet exchange," in Computer Aided Modeling and Design of Communication Links and Networks (CAMAD), 2012 IEEE 17th International Workshop, pp. 216-220, 2012.

[5] Cuzanauskas T., et al., "Activity dependent power allocation algorithm for IEEE 802.11 networks," in Wireless Communications and Mobile Computing Conference (IWCMC), 2015 International, pp. 79-83, 2015.

[6] Ambigavathi M. and Sridharan D., "Priority based AODV routing protocol for critical data in Wireless Body Area Network," in Signal Processing, Communication and Networking (ICSCN), 2015 3rd International Conference, pp. $1-5,2015$.

[7] Dhaya R., et al., "Finest power efficient steering Algorithm for Wireless Sensor Networks for surveillance," in Intelligent Systems and Control (ISCO), 2015 IEEE 9th International Conference, pp. 1-6, 2015.

[8] Liu T., et al., "An improved energy-saving routing protocol for wireless ad hoc networks," in Communication Software and Networks (ICCSN), 2015 IEEE International Conference, pp. 250-254, 2015. 